\title{
Lymph Node Afferent Vessel
}

National Cancer Institute

\section{Source}

National Cancer Institute. Lymph Node Afferent Vessel. NCI Thesaurus. Code C33026.

Lymphatic vessels that transport unfiltered lymph to the lymph nodes. 